\title{
Age-period-cohort analysis of asthma, allergic rhinitis, and atopic dermatitis prevalence in Japan
}

\author{
Tasuju Okuii ${ }^{1^{*}(\mathbb{D})}$ \\ ${ }^{1}$ Medical Information Center, Kyusyu University Hospital, Fukuoka city, Japan \\ *Correspondence: task10300@gmail.com
}

Received: May 13, 2020 Accepted: July 8, 2020

\begin{abstract}
This study aims to analyze the trends in the Japanese prevalence of asthma, allergic rhinitis, and atopic dermatitis by using age-period-cohort (APC) analysis. Data regarding the prevalence of diseases from 1999 to 2017 were collected from Patient Survey in Japan. The data were divided according to age groups ranging from 0-4 years old up to 65-69 years old in 5-year increments. A cohort was defined for each age group of each year with a one-year shift, and cohorts born from 1930-1934 up to 2013-2017 were examined. We used Bayesian APC analysis to decompose the changes in prevalence into age, period, and cohort effects. Results show that the period effect for asthma began to increase in 2008, and those of allergic rhinitis and atopic dermatitis began to increase in 1999. The cohort effects for asthma and atopic dermatitis increased rapidly in cohorts born from approximately 1950 to 1980 and then decreased thereafter. Furthermore, the cohort effect for allergic rhinitis increased from cohorts born in approximately the late 1970s for men and in 1990 for women. The time points with increasing cohort effects for asthma and atopic dermatitis are consistent with the history of air pollution accompanied by rapid economic growth in Japan. The onset of the increased cohort effect for allergic rhinitis was also relatively consistent with the time point at which the mass scattering of pollen began.
\end{abstract}

Keywords: Age-period-cohort analysis, Allergic rhinitis, Asthma, Atopic dermatitis, Prevalence, Air pollution

\section{Introduction}

The number of patients with allergic diseases in Japan has demonstrated an increasing trend in recent years [1]. The estimated number of patients with asthma increased from 1.10 million in 1999 to 1.12 million in 2017, the number of patients with atopic dermatitis increased from 0.40 million to 0.51 million, and the number of patients with allergic rhinitis increased from 0.47 million to 0.66 million. The social burden of allergic diseases is not only limited to the medical expenditures of the diseases but also includes the decrease in a patient's quality of life and labor productivity [2,3]. Therefore, it is important to assess the cause of these increases in allergic diseases and to find methods to prevent them. The representative allergic diseases (i.e., asthma, allergic rhinitis, and atopic dermatitis) are associated with each other, and the comorbidity of these diseases in a patient is relatively common [4]. After developing atopic dermatitis, an infant often develops asthma and allergic rhinitis. This phenomenon is known as atopic march [5]. Environmental factors, such as air pollutants, are believed to be the major cause for allergic diseases [6]. Yokkaichi asthma, which was caused by pollution from petrochemical complexes, emerged in Japan in the 1960s [7], and the massive increase in asthma patients became a social problem. Even in recent years, the association of allergic diseases and particulate matter 2.5 (PM 2.5) or Asian desert dust has gained significant attention in Japan [8]. Exposure to these environmental factors changes depending on the period or birth cohort, and the trend of disease prevalence is considered different not only in terms of periods but also in terms of birth cohorts.

Age-period-cohort (APC) analysis is often used as an analysis method for investigating the trend of disease prevalence [9]. By using APC analysis, we can identify the age, period, and cohort effects on the prevalence of a disease and assess the change of each effect over specified time points. Regarding APC analysis for allergic diseases, APC analysis for asthma has been conducted in some countries [10]. Although APC analysis for childhood asthma had been conducted in Japan [11], the analyzed period was from 1984 to 2004 . Therefore, the exact trend of childhood asthma in recent years is uncertain. The cohorts analyzed in this previous study were also relatively limited because the age period of childhood is short. Although the age of onset for the diseases is high in childhood, we can assess not only the trend of prevalence for older age groups but also the trend of broader birth cohorts by analyzing the data of broader age categories. Data from the Patient Survey in 
Japan are useful because it is a nationwide survey that investigated the number of patients for specific diseases and can be used to assess the trend of allergic diseases. In the current study, we investigated the trend in the prevalence of asthma, allergic rhinitis, and atopic dermatitis in Japan.

\section{Methods}

The data of the Patient Survey in Japan [1] was used for APC analysis. The survey was conducted for grasping the number of patients, and it tallied the number of patients who visit a hospital or a clinic at Japan every three years. The hospitals and clinics that were surveyed were randomly chosen, whereas all hospitals with more than 500 beds were completely surveyed [12]. We used the data of the estimated total number of patients from the seven Patient Surveys conducted from 1999 to 2017. The estimated total number of patients was calculated in the Survey as follows: [12]

(Total number of patients $)=$ (number of hopitalized patients $)+($ number of first visit outpatients $)+$ (number of returning outpatients) $\times$ (mean consultation interval) $\times$ (adjustment factor)

Diseases were classified based on ICD-10 codes in the Survey, and the ICD-10 for the allergic diseases are as follows: J45-J46 for asthma; L20 for atopic dermatitis; and J30 for allergic rhinitis.

We calculated the prevalence of each allergic disease per 1,000 people for men and women from 1999 to 2017 . The prevalence was obtained by dividing the number of patients by total population in Japan by age groups and years. The data of total population was extracted from the data of the Vital Statistics in Japan [13]. The age groups were defined from 0-4 years to 65-69 years by 5-year increments in the data. Also, we used a Bayesian APC model assuming that an outcome follows Poisson distribution for the analysis [9]. The estimated number of a disease was used as outcome value, and corresponding total population was used as offset term. A first-order correlated random-walk was used as the Bayesian prior distributions for each effect. Additionally, in the APC analysis, those who were 65-69 years old in 1999 (i.e., those who were born in 1930-1934) were the first cohort, as this was the oldest birth cohort in the data set. Through a 1-year shift of the birth years, starting from the first cohort, the age group of 0-4 years in 2017 (i.e., those who were born in 2013-2017) was the last cohort. We used the Hamiltonian Monte Carlo method to estimate the parameters [14]. All statistical analyses were conducted using $\mathrm{R}$ version 3.5.1 software [15].

\section{Results}

Figure 1 shows the prevalence of each allergic disease per 1000 people for men and women from 1999 to 2017. Although the prevalence of asthma among men increased in the zero- to four-year-old age group from 1999 to 2017, the prevalence decreased in many other age groups. For women, the prevalence of asthma increased in most age groups, particularly in the zero- to four-year-old age group from 1999 to 2017. Regarding atopic dermatitis, the prevalence among men increased in most age groups from 1999 to 2017. For women, the prevalence increased in all age groups, except for the 15- to 29-yearold age groups over the years. The prevalence for allergic rhinitis increased in most age groups over the years for both men and women.

Figure 2 shows the results of the APC analysis for asthma, atopic dermatitis, and allergic rhinitis for men. The age effect for asthma rapidly decreased until 20 to 24 years and then increased steadily thereafter. The period effect for asthma decreased until 2008 and moved toward an increasing trend thereafter. The cohort effect increased from cohorts born in the early 1950s to the late 1970s and then decreased thereafter. The age effect for atopic dermatitis decreased with an increase in age. The period effect for atopic dermatitis consistently increased from 2002. The cohort effect increased, particularly for cohorts born in the early 1950s to the early 1980s, and then decreased thereafter. The turning point of decrease was the same as that for asthma. The peak of the age effect for allergic rhinitis was five to nine years old. The period effect increased beginning in 2002, and the cohort effect began to increase in cohorts born in approximately 1990.

Figure 3 shows the results of the APC analysis for asthma, atopic dermatitis, and allergic rhinitis for women. Although the degree of decrease in the period effect for asthma was smaller for women than for men until 2008, the degree of increase in the effect beginning in 2008 was larger for women. Although there were subtle differences between sexes, the trends of each effect for atopic dermatitis and allergic rhinitis were relatively similar among men and women.

\section{Discussion}

Regarding asthma, the age effect showed a slight increasing trend with an increase in age from 20-24 years old, and the prevalence increased in older ages. It is known that the age of onset for asthma is also high in older ages [16], and our results seem to show that prevalence increases in older people. Regarding the period effect for asthma, we observed a 

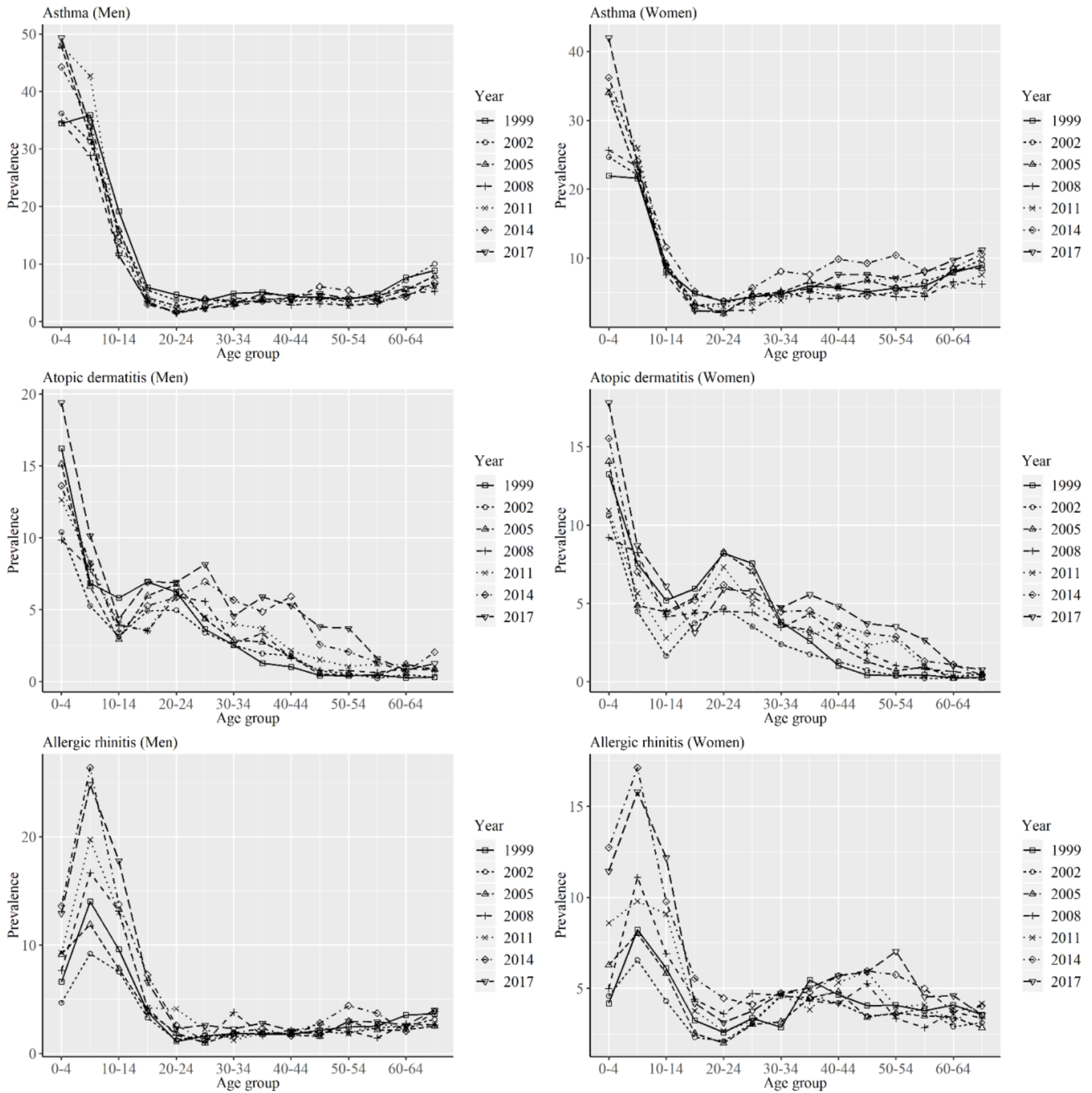

Figure 1. Yearly prevalence of asthma, atopic dermatitis, and allergic rhinitis per 1000 people for age groups among men and women from 1999 to 2017.

difference in the effects among the sexes in terms of the degree of decrease until 2008.

One possible reason for this trend is the rapid decrease of smoking prevalence in men. Smoking prevalence in Japan is higher for men than for women, and smoking prevalence in men has been decreasing in recent years [17]. Smoking is a risk factor for asthma [18], and a decrease in the smoking rate might have contributed to the decrease of the effect. On the other hand, the degree of increase of the period effect from 2008 was larger for women, and the increase was observed in the middle or older age groups (Figure 1). The risk factors for asthma that are associated with adults include stress and alcohol drinking $[19,20]$. Diseases that are associated with stress, such as depression or anxiety disorders, have increased over the years [1], and the degree of increase was larger for women. Furthermore, from 2003 to 2017, the prevalence of alcohol drinking decreased for men while increased for women[21]. Therefore, an increase in psychological stress and the prevalence of alcohol drinking in adults might have led to the increase in the prevalence of asthma, particularly for women. Regarding the cohort effect, the effect showed increasing and decreasing trends. Although there are patients who develop asthma as adults, the age of onset is particularly high in childhood, as shown by our results. Therefore, the cohort effect is 

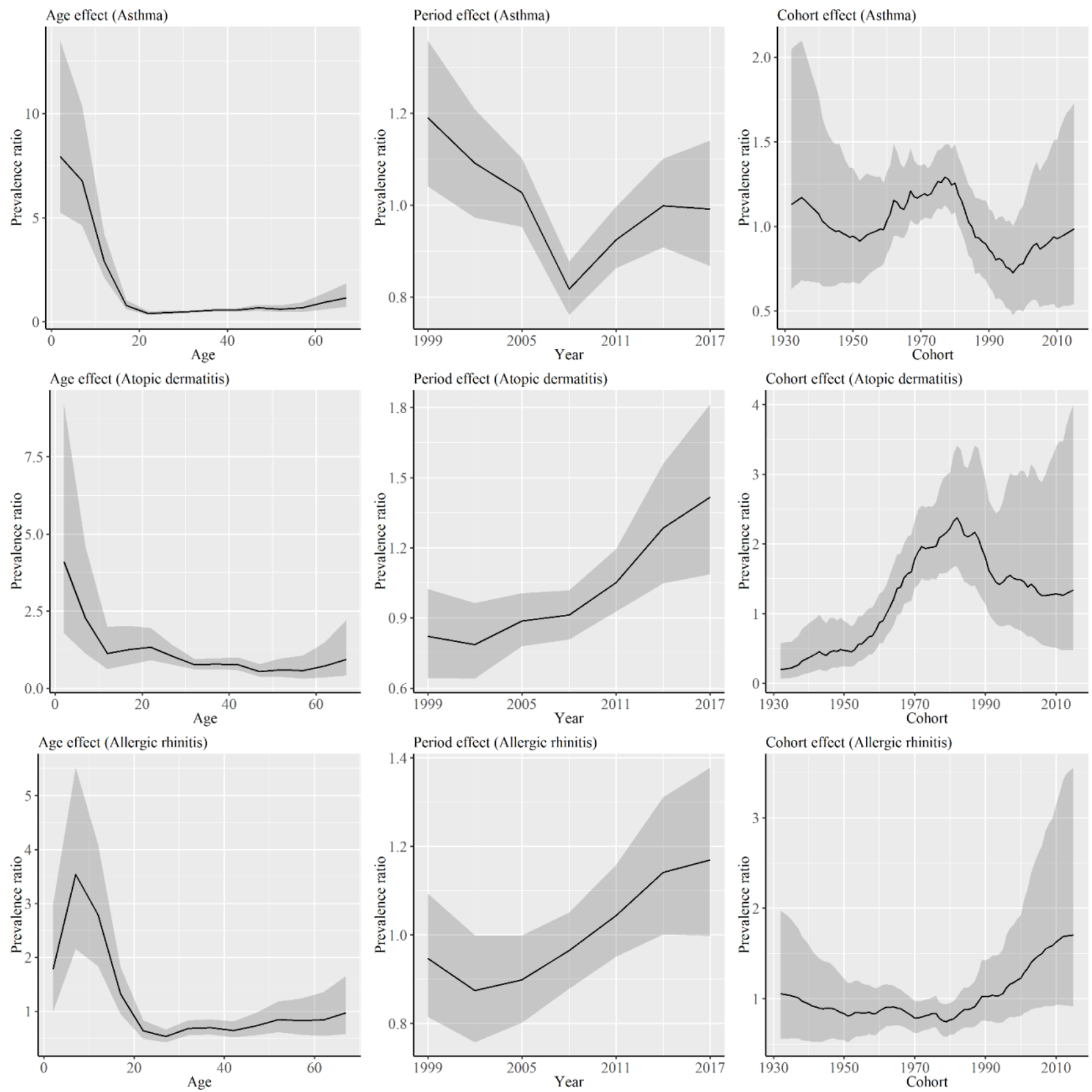

Figure 2. Results of the APC analysis of asthma, atopic dermatitis, and allergic rhinitis for men. Solid lines signify estimates of each effect, and the shadings show $95 \%$ credible intervals of each effect.

considered to reflect the exposure to the risk factors of asthma, particularly in childhood. One possible factor that influences the cohort effect is air pollutants. The cohort effect began to increase from cohorts born in approximately 1950 for both men and women (Figures 2 and 3). At that time, Japan experienced rapid economic growth after World War II. A large-scale outbreak of asthma caused by a petrochemical complex (i.e., Yokkaichi asthma) occurred in the 1960s [7], and air pollution became a social problem in Japan in 1970. China also experienced similar levels of air pollution and an increase in asthma patients during a period of economic growth [22]. The economic growth of Japan is considered related to the increase of the cohort effect. The regulation of exhaust gas from motor vehicles [23] or the revision of environmental standards for nitrogen dioxide [24] began in 1978. These regulations for air pollution are considered to have affected the decrease of the cohort effect for cohorts born in approximately 1980.

The period effect for atopic dermatitis demonstrated an increasing trend from 2002. One possible cause is an increase in the population of urban areas in Japan. There are some reports that have shown the associations between urbanization and the increase in atopic dermatitis [25]; this led to the belief that air pollution in urban areas might affect 

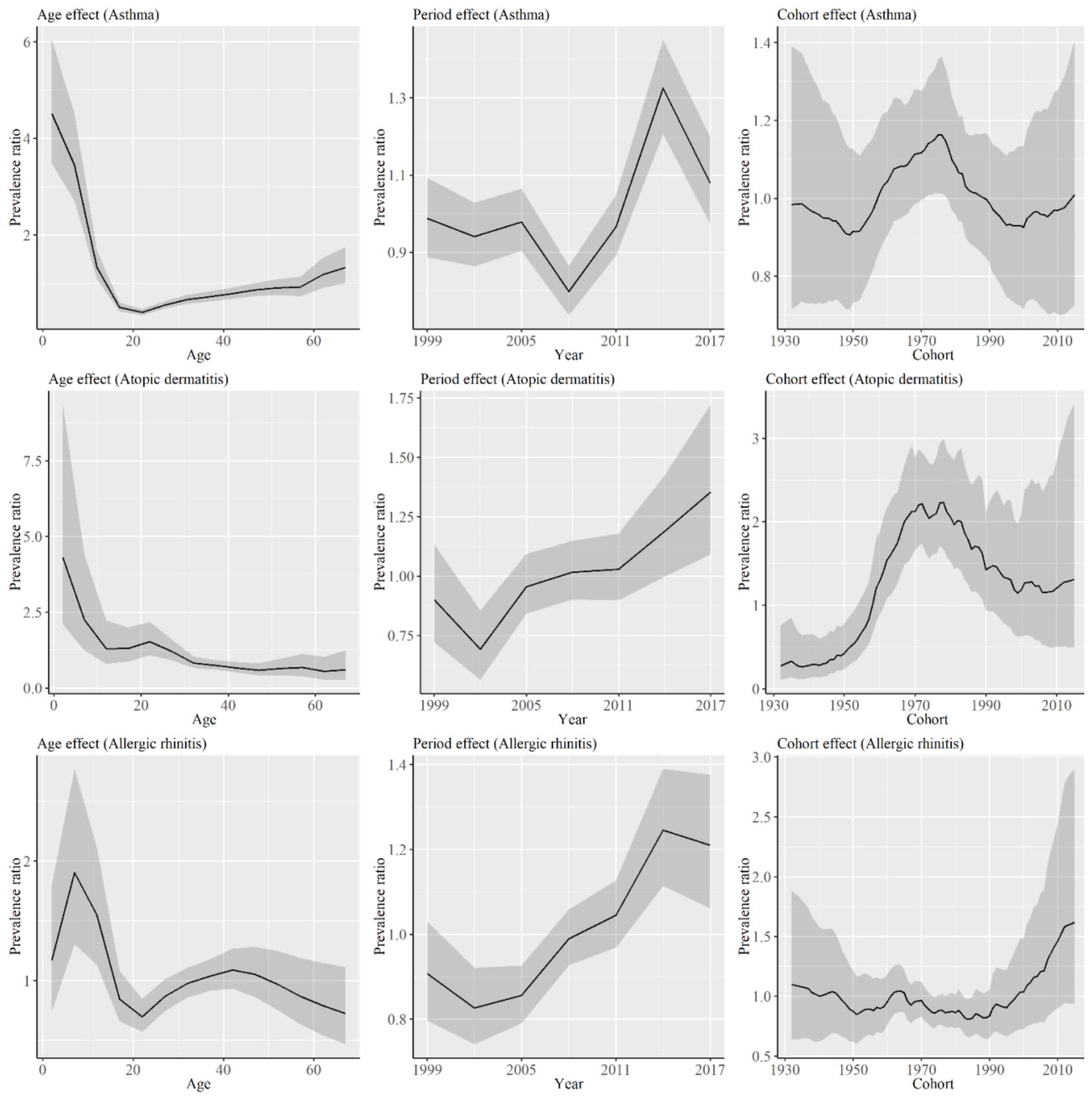

Figure 3. Results of the APC analysis of asthma, atopic dermatitis, and allergic rhinitis for women. Solid lines signify estimates of each effect, and the shadings show $95 \%$ credible intervals of each effect.

disease development. The cohort effect increased from cohorts born in approximately 1950 to 1980, similar to that of asthma. This is considered related to the emissions of air pollutants accompanied by economic growth.

The period effect for allergic rhinitis also increased beginning in 2002. The development of allergic rhinitis is also high in urban areas [26], and urbanization is one possible cause of this increase. Hay fever is the most common type of allergic rhinitis in Japan, and it has been reported that the scattering amount of pollen has increased in Tokyo over the years [27]. The cohort effect increased from cohorts born in approximately 1980 for men and in the 1980s for women. In the 1950s and 1960s, many Japanese cedar and cypress trees were planted on Japanese islands [28]. This is believed to be the main cause of the increase in allergic rhinitis in Japan, particularly hay fever. The mass scattering of pollen occurs from Japanese cedar trees that are 30 years old and older [29], and this is thought to be related to the trend of the cohort effect. The number of patients with hay fever rapidly increased from the late 1970s to 1980 [30].

Although we used nationwide government statistics data, one limitation of this study is that estimates of the number of patients was used for the analysis. To accurately assess the prevalence of these diseases, an epidemiological study that 
targets a broader range of age groups should be conducted.

\section{Conclusions}

We found that the prevalence of asthma, atopic dermatitis, and allergic rhinitis were different depending on the cohorts. The cohort effects for asthma and atopic dermatitis increased rapidly for cohorts born in approximately 1950 to 1980, and the cohort effects for allergic rhinitis consistently increased beginning around the 1980s. An increase in air pollutants accompanied by rapid economic growth and afforestation in the late 20th century appears to be related to the trends of the cohort effects of the analyzed allergic diseases.

\section{Acknowledgement}

Enago has proofread the manuscript.

\section{Conflict of interest}

The authors declare no conflicts of interest associated with this manuscript

\section{ORCID}

Tasuju Okuii: 0000-0001-5098-8502

\section{References}

[1] Ministry of Health, Labour and Welfare of Japan. The Patient Survey. [cited 2020 May 9]. Available from: https://www.estat.go.jp/stat-search/files?page $=1 \&$ toukei=00450022\&tstat=000001031167. (Japanese)

[2] Yaghoubi M, Adibi A, Safari A, FitzGerald JM, Sadatsafavi M. The projected economic and health burden of uncontrolled asthma in the United States. Am J Respir Crit Care Med 2019;200(9):1102-1112.

[3] Drucker AM, Wang AR, Li WQ, Sevetson E, Block JK, Qureshi AA. The burden of atopic dermatitis: summary of a report for the national Eczema association. J Invest Dermatol 2017;137(1):26-30.

[4] Hong S, Son DK, Lim WR, Kim SH, Kim H, Yum HY, et al. The prevalence of atopic dermatitis, asthma, and allergic rhinitis and the comorbidity of allergic diseases in children. Environ Health Toxicol 2012;27:e2012006.

[5] Spergel JM. From atopic dermatitis to asthma: the atopic march. Ann Allergy Asthma Immunol 2010;105(2):99-117.

[6] Takano H, Inoue KI. Environmental pollution and allergies. J Toxicol Pathol 2017;30(3):193-199. (in Japanese)

[7] Kitagawa T. Cause analysis of the Yokkaichi asthma episode in Japan. J Air Pollut Control Assoc 1984;34(7):743-746.

[8] Mimura T, Ichinose T, Yamagami S, Fujishima H, Kamei Y, Goto S, et al. Airborne particulate matter (PM2.5) and the prevalence of allergic conjunctivitis in Japan. Sci Total Environ 2014;487:493-499.

[9] Smith TR, Wakefield J. A Review and Comparison of Age-Period-Cohort Models for Cancer Incidence. Stat Sci 2016; 31(4):591-610.

[10] Pesce G, Locatelli F, Cerveri I, Bugiani M, Pirina P, Johannessen A, et al. Seventy years of asthma in Italy: age, period and cohort effects on incidence and remission of self-reported asthma from 1940 to 2010. PLoS One 2018;13(1):e0191589.

[11] Okamoto E, Hata E, Kobayashi M, Hayashi K. Age-period-cohort analysis of asthma prevalence among school children. Environ Health Prev Med 2007;12(3):119-128.

[12] Ministry of Health, Labour and Welfare of Japan. Overview of the patient survey in 2017. [cited 2020 May 9]. Available from https://www.mhlw.go.jp/toukei/saikin/hw/kanja/17/dl/kanja.pdf. (Japanese)

[13] Ministry of Health, Labour and Welfare of Japan. The vital statistics. [cited 2020 May 9]. Available from: https://www.estat.go.jp/stat-search/files?page=1\&layout=

datalist\&toukei $=00450011 \&$ tstat $=000001028897 \&$ cycle $=7 \&$ tclass $1=000001053058 \&$ tclass $2=000001053061 \&$ tclass $3=000$ 001053072. ( Japanese) 
[14] Stan Development Team. RStan: the R interface to Stan. R package version 2.19.2 [cited 2020 May 9]. Available from: http://mc-stan.org/.

[15] R Core Team: R: A language and environment for statistical computing. R Foundation for Statistical Computing, Vienna, Austria [cited 2020 May 9]. Available from: https://www.R-project.org/.

[16] Fukutomi Y, Taniguchi M, Watanabe J, Nakamura H, Komase Y, Ohta K, et al. Time trend in the prevalence of adult asthma in Japan: findings from population-based surveys in Fujieda City in 1985, 1999, and 2006. Allergol Int. 2011;60(4):443-448.

[17] Funatogawa I, Funatogawa T, Yano E. Trends in smoking and lung cancer mortality in Japan, by birth cohort, 19492010. Bull World Health Organ. 2013;91332-340.

[18] McLeish AC, Zvolensky MJ. Asthma and cigarette smoking: a review of the empirical literature. J Asthma 2010;47(4):345-361.

[19] Lieberoth S, Backer V, Kyvik KO, Skadhauge LR, Tolstrup JS, Grønbæk M, et al. Intake of alcohol and risk of adultonset asthma. Respir Med 2012;106(2):184-188.

[20] Rod NH, Kristensen TS, Lange P, Prescott E, Diderichsen F. Perceived stress and risk of adult-onset asthma and other atopic disorders: a longitudinal cohort study. Allergy 2012;67(11):1408-1414.

[21] Ministry of Health, Labour and Welfare of Japan. the National Health and Nutrition Survey in Japan 2017 [cited 2020 May 9]. Available from: https://www.e-stat.go.jp/stat-search/files?page=1\&toukei=00450171\&tstat=000001041744. (Japanese)

[22] Huang C, Liu W, Hu Y, Zou Z, Zhao Z, Shen L, et al. Updated prevalences of asthma, allergy, and airway symptoms, and a systematic review of trends over time for childhood asthma in Shanghai, China. PLoS One 2015;10(4):e0121577.

[23] Ministry of Environment of Japan. [cited 2020 May 9]. Available from: https://www.env.go.jp/hourei/01/000062.html (Japanese)

[24] Bauner D. International private and public reinforcing dependencies for the innovation of automotive emission control systems in Japan and USA. Transportation Research Part A: Policy and Practice, 2011,45(5),375-388.

[25] Nicolaou N, Siddique N, Custovic A. Allergic disease in urban and rural populations: increasing prevalence with increasing urbanization. Allergy 2005;60(11):1357-1360.

[26] Christensen SH, Timm S, Janson C, Benediktsdottir B, Forsberg B, Holm M, et al. A clear urban-rural gradient of allergic rhinitis in a population-based study in Northern Europe. Eur Clin Respir J 2016;3(1):33463.

[27] Tokyo Metropolitan Government Bureau of Social Welfare and Public Health. Report on actual condition of patients with hay fever. [cited 2020 May 9]. Available from: http://www.tokyoeiken.go.jp/files/kj_kankyo/kafun/jittai/houkokusho.pdf (Japanese)

[28] Yamamoto N, Nishikawa J, Sakamoto M, Shimizu T, Matsuki H. Indoor and outdoor concentrations of Japanese cedar pollens and total suspended particulates: A case study at a kindergarten in Japan, Build Environ 2010;45(3):792-797.

[29] Kuribayashi T, Cossu D, Momotani E. Seroprevalence of Immunoglobulin E Antibodies against Japanese Cedar Pollen Allergens Cry j 1 and Cry j 2 in Dogs Bred in Japan. Vet Sci 2018;5(3):79.

[30] Asakura K. Regional Characteristics of Allergic Rhinitis. Arerugi. 2006;55(11):1390-1393. (Japanese) 